the cis- and trans-regulatory elements that contribute to CD154 gene expression. We have recently identified a NFKB-responsive transcriptional enhancer element located within and just downstream of the 3' CD154 untranslated region. Moreover, using nuclei from primary human CD4 T cells, we have identified a novel DNase I hypersensitive site located approximately $1 \mathrm{~kb}$ upstream of the proximal promoter. This novel site is flanked by several potential $\mathrm{T}$ cell transcription factor binding sites and, thus, may function as a transcriptional enhancer. We are currently comparing the transcriptional activities of these various CD154 cis-regulatory elements in T cells from SLE patients and controls in order to identify region (s) that contribute to enhanced CD154 expression in SLE. Ultimately, we hope to be able to target the cis- or trans-regulatory elements that lead to the abnormal expression of CD154 in SLE and various other autoimmune disorders.

\section{OP0140 CTLA4IG SUBSTANTIALLY AUGMENTS THE BENEFITS OF CYCLOPHOSPHAMIDE FOR LUPUS NEPHRITIS}

P Kaufman, DI Daikh. VA Medical Center and UCSF, San Francisco, CA, USA

10.1136/annrheumdis-2001.73

We have recently shown that combining CTLA4Ig with cyclophosphamide (CTX) results in a marked reversal of active lupus nephritis in NZB/NZW mice. To determine whether similar benefit might be obtained with less CTX, we have compared the effect of a single dose of CTX combined with CTLA4Ig with that of continuous CTX and CTLA4Ig administration. Female mice were treated with either continuous CTX $(50 \mathrm{mg} / \mathrm{kg}$ every 10 days $\times 7$ weeks), continuous CTLA4Ig (50 mg ip $3 \times /$ week $\times 8$ weeks), a single ip injection of CTX $(50 \mathrm{mg} / \mathrm{kg})$ plus brief CTLA4Ig $(50 \mathrm{mg} / \mathrm{kg}$ ip $3 \times /$ week x 2 weeks), a single dose of cyclophosphamide $(50 \mathrm{mg} / \mathrm{kg}$ ip) plus continuous CTLA4Ig, continuous CTX + CTLA4Ig, or saline. After 8 weeks, none of the treated mice had died, while $36 \%$ of the saline group had significant proteinuria and $18 \%$ had died. Mice that had received either continuous CTX, or a combination of prolonged CTLA4Ig with either prolonged or brief CTX had preserved kidney function $(>50 \%$ vs $<10 \%)$. However, survival in the CTX alone group was reduced compared with either the continuous CTX+CTLA4Ig or the single dose CTX+continuous CTLA4Ig groups (73\% vs $100 \%)$. In a subsequent experiment, NZB/NZW mice with either mild of advanced renal disease were treated with low dose CTX $(5 \mathrm{mg} / \mathrm{kg})$ or high dose CTX $(50 \mathrm{mg} / \mathrm{kg})$ in combination with CTLA4Ig. Although mice with advanced renal disease that received either low dose or high dose CTX plus CTLA4Ig had a reduction in proteinuria compared to controls, the improvement was significantly greater among mice receiving the higher dose of CTX. However, among mice with mild disease, the delay in progression of proteinuria was equivalent in mice that received either low dose or high dose CTX in combination with CTLA4Ig. These results indicate that the benefits of CTX in murine lupus nephritis are amplified or sustained by CTLA4Ig, and that the clinical benefits of combining these agents might be realised while at the same time reducing exposure to CTX.

\section{OP0141 ACTIVATED FIBROBLAST-LIKE CELLS IN ASEPTIC PROSTHESIS LOOSENING - SIMILAR OR DIFFERENT FROM RHEUMATOID ARTHRITIS SYNOVIAL FIBROBLASTS?}

${ }^{1,2} \mathrm{~T}$ Pap, ${ }^{3,2} \mathrm{2}^{5}$ Ohtsu, ${ }^{2,4} \mathrm{Y}$ Shigeyama, 5 Sainsbury, ${ }^{2} \mathrm{JK}$ Franz, ${ }^{5} \mathrm{JK}$ Fernihough, ${ }^{5} \mathrm{M}$ Billingham, ${ }^{6} \mathrm{G}$ Pap, ${ }^{6} \mathrm{~W}$ Neumann, ${ }^{2} \mathrm{RE}$ Gay, ${ }^{7} \mathrm{WK}$ Aicher, ${ }^{2} \mathrm{~S}$ Gay. ${ }^{1}$ Rheumatology Research Laboratory, University Hospital Magdeburg, Magdeburg, Germany; ${ }^{2}$ Center of Experimental Rheumatology, Department Rheumatology, University Hospital Zurich, Switzerland; ${ }^{3}$ Department of Orthopaedic Surgery, Tohoku University, Sendai, Japan; ${ }^{4}$ Department of Orthopaedic Surgery, Okayama University, Japan; ${ }^{5}$ OSCOR Facility, University Vet School, Bristol, UK; ${ }^{6}$ Department of Orthopaedic Surgery, University Hospital Magdeburg, Germany; ' Basic Science Research Laboratory, Center of Orthopaedic Surgery, University Hospital Tubingen, Germany

\subsection{6/annrheumdis-2001.74}

Aseptic prosthesis loosening (APL) is an active process that is linked to the development of a synovial-like interface membrane (SLIM) at the bone-implant interface. Growing evidence suggests that the synovial membrane in rheumatoid arthritis (RA) and this SLIM share certain similarities. However, the mechanisms of SLIM formation as well as the cellular and molecular events in the activation of fibroblast-like cells remain elusive. Recently, we have established a novel animal model of SLIM formation that is based on the implantation of joint arthroplasties into intracranially self-stimulated (ICSS) Wistar rats. Data from this animal model as well as from human SLIM samples suggest that there is a subset of activated fibroblast-like cells present also in the SLIM of APL. These prosthesis-loosening fibroblasts (PLF) not only show a similar growth pattern as RA-synovial fibroblasts (RA-SF) but also exhibit features of cellular activation found in RA. Among them, the up regulation of transcription factors and the resistance against Fas-induced apoptosis are most prominent. Although PLF express a number of matrix degrading enzymes and secrete soluble factors that stimulate neighbouring cells, their role in the degradation of bone has been restricted to that of supporting bystanders. Based on most recent results, we report that PLF from the SLIM around loose joint prostheses display a highly aggressive, RA-like phenotype when implanted into SCID mice, are capable of resorbing bone in vivo without the help of osteoclasts and release large amounts of acidic components. As demonstrated by RT-PCR, in situ hybridization and immuno electron microscopy, these cells express a specific proton pump (v-ATPase) on their cell surface that may account for their ability to decalcify bone. By quantitative real time PCR, an increased expression of this $\mathrm{v}$-ATPase is found in prosthesis loosening fibroblasts and rheumatoid synovial fibroblasts as compared to normal or osteoarthritic cells. Inhibition of the $\mathrm{v}$ ATPase with specific inhibitors results in a decrease of pericellular acidification. Investigating the exon structure of its catalytic $\beta 1$ subunit, we were able to describe a hitherto unknown vATPase splice variant that appears to be regulated differently in prosthesis loosening fibroblasts as compared to rheumatoid and osteoarthritic cells. The expression of specific v-ATPases on their cell membranes including novel splice variants may provide the means by which these cells are able to decalcify bone prior to its degradation. Further studies will have to clarify whether the capability of PLF to resorb bone constitutes a rather unique feature of these cells or is linked also to cellular activation in RA. 